\title{
Efeito de Espalhante-Adesivo na Eficiência dos Acaricidas Propargite e Cyhexatin no Controle do Brevipalpus phoenicis (Geijskes) (Acari: Tenuipalpidae) em Citros
}

\author{
Carlos A. L. Oliveira ${ }^{1}$, Tomomassa Matuo ${ }^{1}$, José E. Santos Jr. ${ }^{1}$ e Mílton C. Toledo ${ }^{1}$ \\ ${ }^{1}$ Departamento de Defesa Fitossanitária da FCAV/UNESP, Rodovia Carlos \\ Tonanni, km 5, 14870-000, Jaboticabal, SP.
}

An. Soc. Entomol. Brasil 26(3): 487-493 (1997)
Effect of Spreader-Sticker on the Efficacy of Mitecides Propargite and Cyhexatin to Control Brevipalpus phoenicis (Geijskes)
(Acari: Tenuipalpidae) on Citrus

\begin{abstract}
A field work was conducted using the mitecides propargite and cihexatin in two formulations (WP and FW) with or without the addition of a spreader-sticker (polyoxyethylene alkylphenol ether), for the control of citrus leprosis mite, Brevipalpus phoenicis (Geijskes). Two field trials were carried out, in 1991 and 1994, in Natal citrus orchards. Spraying was performed using a tractor powered high pressure spray gun using 18 l and 11 l of spray solution/ tree, respectively. Effect of the spreader-sticker was evaluated counting the number of mites before and after treatments. Retention of the spray liquid on the leaves was not affected by the addition of spreader-sticker. Reduction up to 95.6\% in mite population was observed with the increase in the dosage rate of the propargite, and up to $98.9 \%$ for the cyhexatin in relation to the lower dosage. Spreader-sticker did not increase the efficiency of the cyhexatin, although the total number of the mites, independently of dosage and formulation was $50.1 \%$ lower in treatments with spreader-sticker.
\end{abstract}

KEY WORDS: Acari, surfactant, spraying, mitecide, formulations.

RESUMO - Embora espalhantes-adesivos sejam considerados importantes em pulverizações, existem dúvidas quanto à sua utilidade em pulverizações a alto volume quando o escorrimento é maior, como nas aplicações com equipamentos tipo pistola, para o controle de Brevipalpus phoenicis (Geijskes). Dois ensaios de campo foram conduzidos, em 1991 e 1994, em pomar de citros cv. Natal. As pulverizações foram realizadas com pulverizador tratorizado equipado com pistolas de alta pressão usando 181 e 111 de calda/planta, respectivamente. Efeitos dos espalhante-adesivo foi avaliado contando-se o número de ácaros antes e após a aplicação. Não se verificaram diferenças sensíveis na retenção do líquido pulverizado nas folhas de citros quando se adicionou o espalhante-adesivo à calda com propargite. Redução de até $95,6 \%$ na população do ácaro foi obsevada com o aumento na dosagem do propargite e de até $98,9 \%$ em relação de menor dosagem para o cyhexatin. Constatou-se que o espalhante-adesivo não melhorou a eficiência do cyhexatin, embora a somatória dos totais de ácaros, independentemente da dosagem e da formulação, tenha sido 50,1\% menor com o uso de espalhante-adesivo.

PALAVRAS-CHAVE: Acari, surfactante, pulverização, acaricidas, formulações. 
Os adjuvantes classificados como espalhantes-adesivos são substâncias empregadas em pulverizações para melhorar a eficiência dos agrotóxicos. Funcionam como agentes redutores da tensão superficial das gotas de pulverização, promovendo por ação interfacial o equilíbrio estável entre as faces de contato, proporcionando espalhamento e adesão das gotas nas folhas, assim como na absorção do produto pela planta (Johnstone 1973). Segundo Ocampo-Ruiz \& Matuo (1994), a necessidade de espalhantes-adesivos torna-se questionável em aplicações a alto volume, onde as gotas individualizadas coalescem na superfície da folha, formando glóbulos maiores que posteriormente escorrem.

Ao estudarem o efeito de adjuvantes na retenção de água sobre folha de laranjeira cv. Natal, Matuo et al. (1989) verificaram que os espalhantes-adesivos proporcionaram uma menor retenção do líquido nas folhas de laranjeira em pulverizações de alto volume. A partir desse fato, surgiu a hipótese de uma menor retenção acarretar uma redução do poder residual do acaricida, afetando sua eficiência. Chiavegato et al. (1993) verificaram que o controle de ovos e adultos de Brevipalpus phoenicis (Geijskes) por hexythiazox não foi afetado pela adição de espalhante-adesivo. A eficiência dos acaricidas-ovicidas hexythiazox e quinometionato em frutos cítricos, com e sem espalhante-adesivo e submetidos à chuva artificial, não foi afetada e o espalhante não os favoreceu (Chiavegato et al. 1994). Os espalhantes testados por Ocampo-Ruiz \& Matuo (1994) diminuíram a tensão superficial e a retenção do propargite pelas folhas pulverizadas em alto volume; porém esta redução não se refletiu no efeito biológico do acaricida no controle do ácaro da leprose dos citros. Não influíram na duração da ação acaricida e nem impediram a lavagem do produto após submetidos a $10 \mathrm{~mm}$ de chuva artificial.

Em razão das dúvidas que pairam sobre o assunto, a pesquisa visou avaliar se espalhantes à base de polioxietileno alquilfenol éter (Hodefix, Haiten, IharaguenS e Citowett 200) apresentam comportamento diferenciado com relação ao propargite e ao cyhexatin nas formulações pó-molhável e suspensão concentrada, no controle do ácaro da leprose, em pulverização em condições de campo na cultura de citros.

\section{Material e Métodos}

Foram conduzidos dois ensaios de campo, em 1991 e 1994, em pomares de citros comerciais da cv. Natal localizados no município de Bebedouro e Viradouro, SP, com plantas de 23 e 5 anos de idade e o espaçamento era de $6 \mathrm{~m} \times 6 \mathrm{~m}$ e $8 \mathrm{mx} 5 \mathrm{~m}$, respectivamente. Os experimentos foram delineados em blocos casualizados, com 9 e 13 tratamentos repetidos 10 e 6 vezes. A adoção deste delineamento foi devido às plantas apresentarem níveis populacionais diferentes do ácaro, avaliados previamente. Cada bloco foi constituído de plantas que apresentavam aproximadamente o mesmo número de ácaros, não se considerando a localização das plantas no pomar. Em cada bloco, sortearam-se as plantas de cada tratamento. Cada parcela experimental constou de uma planta distante de pelo menos uma planta da parcela adjacente.

No teste 1, desenvolvido em 1991, utilizou-se o propargite (Omite 720 BR CE), nas dosagens de $25,50,75$ e $100 \mathrm{ml}$ p.c. $/ 100$ 1 de água, com e sem espalhante-adesivo (Haiten), a $10 \mathrm{ml} / 1001$ de água. No teste 2, conduzido em 1994 utilizou-se o cyhexatin (Sipcatin 500 SC e Hokko Cyhexatin 500 $\mathrm{PM}$ ), nas dosagens de 12,5, 25,0 e 50,0 ml ou g de p.c./100 l de água, com e sem espalhante adesivo (Hodefix), a $15 \mathrm{ml}$ por 1001 de água.

As aplicações foram realizadas em 27/06/ 91 (teste 1) e 21/6/94 (teste 2), utilizando-se um pulverizador tratorizado tipo pistola, dotado de bico D6, tracionado por trator e regulado a uma pressão de $2.070 \mathrm{kPa}(300$ $\mathrm{lbf} / \mathrm{pol}^{2}$ ). Foram gastos $18 \mathrm{l}$ da calda por planta no teste 1 e 111 da calda por planta no teste 2 . No teste 1 foram realizados levantamentos populacionais do ácaro da leprose um dia 
antes da aplicação, e 7, 14, 21, 28, 41, 55, 70, 83 e 97 dias após a aplicação dos produtos. No teste 2, foi realizado levantamento populacional um dia antes da aplicação, e 9, 17, 38, 50, 66 e 93 dias após a aplicação. Em cada parcela, foram coletados ao acaso 10 frutos com sintomas de verrugose ao redor da copa das árvores por ocasião dos levantamentos (Martinelli et al. 1976). As amostras de cada parcela foram acondicionadas separadamente em sacos de papel e levadas ao laboratório para avaliação. Para tanto, procedeu-se à retirada dos ácaros dos frutos através de uma máquina de varredura. Os ácaros foram contados com auxílio de um microscópio estereoscópico em uma área de $12 \mathrm{~cm}^{2}$ da placa de vidro do aparelho (Oliveira 1983). Os dados observados nos levantamentos foram transformados em $\log (\mathrm{x}+$ $1,0)$ e $\log (x+1,5)$ e analisados pelos testes $F$ e Tukey, nos esquemas fatoriais $4 \times 2+$ testemunha (teste 1 ) e $2 \times 3 \times 2+$ testemunha (teste 2).

Em laboratório, determinou-se a retenção da pulverização nas folhas cítricas segundo a metodologia descrita por Matuo et al. (1989). Para tanto, uma folha mantida na posição vertical e apoiada em um suporte colocado sobre o prato de uma balança com precisão de $1 \mathrm{mg}$ foi pulverizada até além do ponto de escorrimento. Após o escorrimento, o peso do líquido retido na superfície da folha foi anotado e expresso em $\mathrm{ml} / \mathrm{m}^{2}$ de folha.

\section{Resultados e Discussão}

Constatou-se em todos os tratamentos com acaricidas reduções significativas nos níveis populacionais durante todo o período da condução do ensaio (Tabela 1). Em quaisquer das dosagens testadas a eficiência do propargite foi a mesma com ou sem a adição de espalhante-adesivo, evidenciando que a eficiência do acaricida não aumentou com a adição do surfactante. Ocorreu um incremento na redução da população do $B$. phoenicis de até $95,6 \%$ com o aumento da dosagem do acaricida em relação a menor.

Os acaricidas foram empregados em subdosagens com o objetivo de constatar possíveis efeitos da adição do espalhanteadesivo sobre a eficiência dos acaricidas, uma vez que, eventuais efeitos de adjuvantes poderiam ser melhor detectados na faixa de dosagem abaixo da convencionalmente recomendada.

Verificou-se que o propargite, com ou sem a adição de espalhante-adesivo, reduziu de 58,2 a $62,7 \%$ a retenção da calda nas folhas quando comparada à água (Tabela 2), confirmando os resultados já relatados por Johnstone (1973), Matuo et al.(1989) e Ocampo-Ruiz \& Matuo (1994), que observaram reduções na retenção do líquido quando se reduz a tensão superficial do mesmo. Notase também que não houve diferença sensível na retenção do líquido pulverizado nas folhas de citros quando se adicionou o espalhanteadesivo à calda acaricida. Tal fato se explica em virtude de o acaricida propargite já possuir em sua formulação componentes com ação surfactante que conferem ao produto características semelhantes às que se obteria quando da adição do espalhante-adesivo. Matuo et al. (1989) observaram que a adição de espalhantes prejudicaram a retenção do propargite pelas folhas de citros e formularam a hipótese de que essa menor retenção poderia diminuir o poder residual do acaricida. Essa hipótese não pode ser confirmada no presente trabalho, uma vez que as retenções com a presença ou ausência do espalhante-adesivo foram semelhantes.

Pelos resultados obtidos no teste 2, constatou-se que o cyhexatin causou reduções das populações do B. phoenicis, cujo efeito se prolongou por todo o período de avaliação (Tabela 3). A interação formulação $x$ espalhante-adesivo não foi significativa em nenhum dos levantamentos efetuados após a aplicação e o cyhexatin, independentemente da formulação, comportou-se de modo semelhante nas várias dosagens testadas, com ou sem a adição do espalhante-adesivo.

Observou-se que a formulação influenciou o desempenho do acaricida somente no levantamento realizado 50 dias após a aplicação, com diferença significativa entre 
Tabela 1. Número total de Brevipalpus phoenicis, varridos de frutos e observados nas placas de contagem, nos levantamentos realizados antes e após a aplicação do propargite, com e sem espalhante-adesivo. Bebedouro, SP, de 26/6/91 a 2/10/91. Teste 1.

\begin{tabular}{|c|c|c|c|c|c|c|c|c|c|c|c|c|}
\hline \multirow{2}{*}{ Tratamentos } & \multirow{2}{*}{$\begin{array}{l}\text { Dosagens }^{1} \\
\text { ml p.c./100 L } \\
\text { água }\end{array}$} & \multicolumn{10}{|c|}{ Dias após a aplicação } & \multirow{2}{*}{ Total } \\
\hline & & Prévia & 7 & 14 & 21 & 28 & 41 & 55 & 70 & 83 & 97 & \\
\hline Testemunha & - & 347 & 144 & 162 & 507 & 228 & 513 & 460 & 239 & 559 & 388 & 3200 \\
\hline Propargite & 25 & 340 & 22 & 9 & 11 & 6 & 149 & 144 & 13 & 180 & 129 & 663 \\
\hline Propargite & 50 & 336 & 2 & 1 & 4 & 2 & 18 & 22 & 6 & 44 & 40 & 139 \\
\hline Propargite & 75 & 338 & 1 & 1 & 1 & 0 & 3 & 6 & 1 & 40 & 16 & 69 \\
\hline Propargite & 100 & 342 & 2 & 3 & 1 & 2 & 13 & 18 & 3 & 27 & 45 & 144 \\
\hline \multicolumn{13}{|l|}{ Propargite +} \\
\hline $\begin{array}{l}\text { Esp.-ades. } \\
\text { Propargite + }\end{array}$ & $25+10$ & 344 & 19 & 10 & 11 & 6 & 56 & 76 & 14 & 173 & 247 & 612 \\
\hline \multicolumn{12}{|l|}{ Propargite + } & 260 \\
\hline $\begin{array}{l}\text { Esp--ades. } \\
\text { Propargite + }\end{array}$ & $75+10$ & 339 & 0 & 0 & 4 & 1 & 10 & 3 & 3 & 28 & 22 & 71 \\
\hline Esp.-ades. & $100+10$ & 343 & 0 & 3 & 1 & 0 & 4 & 2 & 2 & 10 & 5 & 27 \\
\hline \multicolumn{13}{|c|}{ Desdobramentos } \\
\hline \multicolumn{12}{|c|}{$0,16 \mathrm{~ns} 260,41 * * 340,69 * * 630,41 * * 691,71 * * 154,05 * * 100,57 * 246,95 * * 53,56 * 57,94 * *$} & $7,94 * *$ \\
\hline \multicolumn{13}{|c|}{ Propargite vs. } \\
\hline Esp. - ades. & & $0,16 \mathrm{~ns}$ & $0,03 \mathrm{~ns}$ & $0,46 \mathrm{~ns}$ & $0,04 \mathrm{~ns}$ & $0,32 \mathrm{~ns}$ & $2,87 \mathrm{~ns}$ & $0,22 \mathrm{~ns}$ & 2,95 & ins $0,02 \mathrm{~ns}$ & 0,0 & Ons \\
\hline
\end{tabular}

${ }^{1}$ p.c. $=$ produto comercial; Propargite $=$ Omite $720 \mathrm{BR}$ CE; Espalhante-adesivo $=$ Haiten.

as formulações, sendo a PM mais eficiente no controle do ácaro da leprose dos citros (Tabela 4). Entretanto, essa diferença desapareceu nos levantamentos posteriores, o que dificulta formular hipóteses sobre esse comportamento. O cyhexatin, na dosagem de $50 \mathrm{~g}$ ou $\mathrm{ml}$ (dosagem de registro), independentemente da formulação, foi mais eficaz no controle do acarino nos levantamentos efetuados 38 e 66 dias após a aplicação quando confrontada a 12,5; porém semelhante à dosagem intermediária $(25,0)$ que, por sua vez, não diferiu da menor .

Observou-se que a adição do espalhanteadesivo ao cyhexatin nas formulações SC e PM não melhorou a ação do produto, embora os dados mostrem uma tendência de maior eficácia do cyhexatin com espalhante-adesivo, independentemente da formulação .

Os espalhantes-adesivos testados por Ocampo-Ruiz \& Matuo (1994) diminuíram a tensão superficial e a retenção do acaricida propargite e não impediram a lavagem do produto após submetido a $10 \mathrm{~mm}$ de água. De acordo com esses autores, líquido com menor tensão superficial é menos retido porque o tamanho dos glóbulos na superfície das folhas é menor em líquido com baixa tensão superficial, pois a película se rompe com maior facilidade e o escorrimento é maior.

Provavelmente a chuva em baixa intensidade, ocorrida no período após a aplicação no teste 2 , não interferiu na eficácia do 
Tabela 2. Quantidade de líquido pulverizado retido pelas folhas de citros, com e sem espalhante-adesivo em laboratório.

\begin{tabular}{lcc}
\hline Tratamentos & $\begin{array}{c}\text { Dosagens }^{1} \\
\text { ml p.c./100 L }\end{array}$ & $\begin{array}{c}\text { Líquido retido pela } \\
\text { superfície das folhas } \\
\left(\mathrm{ml} / \mathrm{m}^{2}\right)-\text { média de } \\
3 \text { determinações }\end{array}$ \\
\hline Propargite & 25 & 79,6 \\
Propargite & 50 & 72,7 \\
Propargite & 75 & 72,0 \\
Propargite & 100 & 71,3 \\
Propargite + Esp.-ades. & $25+10$ & 72,1 \\
Propargite + Esp.-ades. & $50+10$ & 71,3 \\
Propargite + Esp.-ades. & $75+10$ & 71,2 \\
Propargite + Esp.-ades. & $100+10$ & 71,0 \\
Água & - & 190,3 \\
\hline
\end{tabular}

${ }^{1}$ p.c. $=$ produto comercial; Propargite $=$ Omite 720 BR CE $(72 \%) ;$ Espalhante-adesivo $=$ Haiten .

cyhexatin em ambas as formulações, uma vez que o uso do surfactante, aparentemente, proporcionou uma melhora na eficiência do acaricida. A interação dosagem x espalhanteadesivo foi significativa nos levantamentos efetuados 50 e 60 dias após a aplicação.

Tabela 3. Totais de Brevipalpus phoenicis,varridos de frutos e observados nas placas de contagem, nos levantamentos realizados antes e após a aplicação do cyhexatin, nas formulações SC e PM, com e sem espalhante-adesivo. Viradouro, SP, de 20/6/94 a 22/9/94. Teste 2.

\begin{tabular}{|c|c|c|c|c|c|c|c|c|c|}
\hline \multirow{2}{*}{ Tratamentos } & \multirow{2}{*}{$\begin{array}{c}\text { Dosagens }^{1} \\
\text { ml ou g pc/ } \\
100 \text { lágua }\end{array}$} & \multirow[b]{2}{*}{ Prévia } & \multicolumn{6}{|c|}{ Dias após a aplicação } & \multirow[b]{2}{*}{ Total } \\
\hline & & & 9 & 17 & 38 & 50 & 66 & 93 & \\
\hline Cyhexatin SC & 12,5 & 194 & 0 & 1 & 24 & 14 & 133 & 77 & 249 \\
\hline Cyhexatin SC & 25,0 & 191 & 0 & 0 & 32 & 12 & 0 & 91 & 135 \\
\hline $\begin{array}{l}\text { Cyhexatin SC } \\
\text { Cyhexatin SC + }\end{array}$ & 50,0 & 195 & 0 & 0 & 0 & 0 & 3 & 23 & 26 \\
\hline & $12,5+15$ & 192 & 4 & 14 & 12 & 25 & 26 & 117 & 198 \\
\hline $\begin{array}{l}\text { Esp.-ades. } \\
\text { Cyhexatin SC + }\end{array}$ & $25,0+15$ & 192 & 3 & 0 & 0 & 8 & 2 & 34 & 47 \\
\hline Esp.-ades. & $50,0+15$ & 195 & 1 & 0 & 1 & 12 & 0 & 3 & 17 \\
\hline Cyhexatin PM & 12,5 & 193 & 11 & 1 & 8 & 3 & 152 & 63 & 238 \\
\hline Cyhexatin PM & 25,0 & 194 & 6 & 3 & 1 & 0 & 0 & 56 & 66 \\
\hline $\begin{array}{l}\text { Cyhexatin PM } \\
\text { Cyhexatin PM + }\end{array}$ & 50,0 & 193 & 3 & 0 & 0 & 0 & 0 & 0 & 3 \\
\hline $\begin{array}{l}\text { Esp.-ades. } \\
\text { Cyhexatin PM + }\end{array}$ & $12,5+15$ & 192 & 21 & 11 & 12 & 5 & 10 & 34 & 93 \\
\hline $\begin{array}{l}\text { Esp.-ades. } \\
\text { Cyhexatin PM + }\end{array}$ & $25,0+15$ & 195 & 6 & 0 & 0 & 2 & 0 & 8 & 16 \\
\hline Esp.-ades. & $50,0+15$ & 197 & 0 & 1 & 0 & 0 & 0 & 0 & \\
\hline Testemunha & - & 196 & 138 & 244 & 252 & 270 & 967 & 1324 & 3195 \\
\hline
\end{tabular}

${ }^{1}$ p.c. $=$ produto comercial; Cyhexatin $\mathrm{SC}=$ Sipcatin $500 \mathrm{SC} ;$ Cyhexatin $\mathrm{PM}=$ Hokko Cyhexatin 500; Espalhante adesivo $=$ Hodefix. 
Tabela 4. Número médio de Brevipalpus phoenicis transformado em $\log (\mathrm{x}+1,5)$ observado nas parcelas tratadas com cyhexatin nas formulações suspensão concentrada (SC) e pó-molhável (PM) nas dosagens de 12,5, 25,0 e 50,0 g ou ml dos produtos comerciais e com e sem espalhanteadesivo nas avaliações efetuadas antes e após 9 a 93 dias da aplicação. Viradouro, SP. 1994. Teste 2 .

\begin{tabular}{|c|c|c|c|c|c|c|c|}
\hline \multirow[t]{2}{*}{ Avaliações } & \multicolumn{2}{|c|}{ Formulações $^{1}$} & \multicolumn{3}{|c|}{ Dosagens $^{1}$} & \multicolumn{2}{|c|}{ Espalhante-adesivo $^{1}$} \\
\hline & $\mathrm{SC}$ & $\mathrm{PM}$ & 12,5 & 25,0 & 50,0 & Sem & Com \\
\hline Prévia & $\begin{array}{c}193,2 \mathrm{a} \\
{[ \pm 0,07](36)}\end{array}$ & $\begin{array}{c}194,0 \mathrm{a} \\
{[ \pm 0,07](36)}\end{array}$ & $\begin{array}{c}192,8 \mathrm{a} \\
{[ \pm 0,01](24)}\end{array}$ & $\begin{array}{c}193,0 \mathrm{a} \\
{[ \pm 0,01](24)}\end{array}$ & $\begin{array}{c}195,0 \text { a } \\
{[ \pm 0,01](24)}\end{array}$ & $\begin{array}{c}193,3 \mathrm{a} \\
{[ \pm 0,07](36)}\end{array}$ & $\begin{array}{c}193,8 \mathrm{a} \\
{[ \pm 0,07](36)}\end{array}$ \\
\hline 9 & $\begin{array}{c}1,3 \mathrm{a} \\
{[ \pm 0,10](36)}\end{array}$ & $\begin{array}{c}7,8 \mathrm{a} \\
{[ \pm 0,10](36)}\end{array}$ & $\begin{array}{c}9,0 \mathrm{a} \\
{[ \pm 0,12](24)}\end{array}$ & $\begin{array}{c}3,8 \mathrm{a} \\
{[ \pm 0,12](24)}\end{array}$ & $\begin{array}{c}1,0 \mathrm{a} \\
{[ \pm 0,12](24)}\end{array}$ & $\begin{array}{c}3,3 \mathrm{a} \\
{[ \pm 0,10](36)}\end{array}$ & $\begin{array}{c}5,8 \mathrm{a} \\
{[ \pm 0,10](36)}\end{array}$ \\
\hline 17 & $\begin{array}{c}2,5 \mathrm{a} \\
{[ \pm 0,07](36)}\end{array}$ & $\begin{array}{c}2,7 \mathrm{a} \\
{[ \pm 0,07](36)}\end{array}$ & $\begin{array}{c}6,8 \mathrm{a} \\
{[ \pm 0,08](24)}\end{array}$ & $\begin{array}{c}0,8 \mathrm{a} \\
{[ \pm 0,08](24)}\end{array}$ & $\begin{array}{c}0,3 \mathrm{a} \\
{[ \pm 0,08](24)}\end{array}$ & $\begin{array}{c}0,8 \mathrm{a} \\
{[ \pm 0,07](36)}\end{array}$ & $\begin{array}{c}4,3 \mathrm{a} \\
{[ \pm 0,07](36)}\end{array}$ \\
\hline 38 & $\begin{array}{c}11,5 \mathrm{a} \\
{[ \pm 0,11](36)}\end{array}$ & $\begin{array}{c}3,5 \mathrm{a} \\
{[ \pm 0,11](36)}\end{array}$ & $\begin{array}{c}14,0 \mathrm{a} \\
{[ \pm 0,14](24)}\end{array}$ & $\begin{array}{c}8,3 \mathrm{ab} \\
{[ \pm 0,14](24)}\end{array}$ & $\begin{array}{c}0,3 \mathrm{~b} \\
{[ \pm 0,14](24)}\end{array}$ & $\begin{array}{c}10,8 \mathrm{a} \\
{[ \pm 0,11](36)}\end{array}$ & $\begin{array}{c}4,2 \mathrm{a} \\
{[ \pm 0,11](36)}\end{array}$ \\
\hline 50 & $\begin{array}{c}11,8 \mathrm{a} \\
{[ \pm 0,09](36)}\end{array}$ & $\begin{array}{c}1,7 \mathrm{~b} \\
{[ \pm 0,09](36)}\end{array}$ & $\begin{array}{c}11,8 \mathrm{a} \\
{[ \pm 0,11](24)}\end{array}$ & $\begin{array}{c}5,5 \mathrm{a} \\
{[ \pm 0,11](24)}\end{array}$ & $\begin{array}{c}3,0 \mathrm{a} \\
{[ \pm 0,11](24)}\end{array}$ & $\begin{array}{c}4,8 \mathrm{a} \\
{[ \pm 0,09](36)}\end{array}$ & $\begin{array}{c}8,7 \mathrm{a} \\
{[ \pm 0,09](36)}\end{array}$ \\
\hline 66 & $\begin{array}{c}27,3 \mathrm{a} \\
{[ \pm 0,14](36)}\end{array}$ & $\begin{array}{c}27,0 \mathrm{a} \\
{[ \pm 0,14](36)}\end{array}$ & $\begin{array}{c}80,3 \mathrm{a} \\
{[ \pm 0,17](24)}\end{array}$ & $\begin{array}{c}0,5 \mathrm{ab} \\
{[ \pm 0,17](24)}\end{array}$ & $\begin{array}{c}0,8 \mathrm{~b} \\
{[ \pm 0,17](24)}\end{array}$ & $\begin{array}{c}48,0 \mathrm{a} \\
{[ \pm 0,14](36)}\end{array}$ & $\begin{array}{c}6,3 \mathrm{a} \\
{[ \pm 0,14](36)}\end{array}$ \\
\hline 93 & $\begin{array}{c}57,5 \mathrm{a} \\
{[ \pm 0,21](36)}\end{array}$ & $\begin{array}{c}26,8 \mathrm{a} \\
{[ \pm 0,21](36)}\end{array}$ & $\begin{array}{c}72,8 \mathrm{a} \\
{[ \pm 0,26](24)}\end{array}$ & $\begin{array}{c}41,3 \mathrm{a} \\
{[ \pm 0,26](24)}\end{array}$ & $\begin{array}{c}6,5 \mathrm{a} \\
{[ \pm 0,26](24)}\end{array}$ & $\begin{array}{c}51,7 \mathrm{a} \\
{[ \pm 0,21](36)}\end{array}$ & $\begin{array}{c}32,7 \mathrm{a} \\
{[ \pm 0,21](36)}\end{array}$ \\
\hline
\end{tabular}

${ }^{1}$ Médias seguidas da mesma letra na linha não diferem estatisticamente entre si pelo teste Tukey $(\mathrm{P} \leq 0,05)$.

Através do desdobramento das médias de dosagens e espalhante-adesivo na avaliação realizada 50 dias após a aplicação, verificouse que os tratamentos com espalhante-adesivo não diferiram estatisticamente para as três dosagens de cyhexatin, enquanto que os tratamentos sem espalhante-adesivo apresentaram diferença estatística significativa. $\mathrm{O}$ cyhexatin, na dosagem de 25,0, apresentou uma eficiência maior sem espalhante quando confrontado ao tratamento com espalhanteadesivo.

Resultados obtidos no levantamento realizado 66 dias após a aplicação, mediante o desdobramento da interação dosagem $x$ espalhante-adesivo mostraram que o cyhexatin, na dosagem de 12,5 , foi beneficiado pela adição do espalhante-adesivo no controle do ácaro, enquanto que, para as demais dosagens, o surfactante em nada contribuiu para melhorar o desempenho do acaricida.
Observou-se, também, que as diferentes dosagens de cyhexatin com espalhanteadesivo não diferiram entre si, enquanto que, sem espalhante, a eficiência foi maior à medida que se aumentou a dosagem. Redução de até $98,7 \%$ na população do ácaro foi observada com o aumento na dosagem de acaricida, independentemente da formulação.

Em vista do exposto, conclui-se que o espalhante-adesivo não melhorou a eficiência do cyhexatin, embora a somatória dos totais de ácaros, independentemente da dosagem e da formulação, tenha sido $50,1 \%$ menor com o uso de espalhante-adesivo.

\section{Literatura Citada}

Chiavegato, L.G., C.E.T. Nogueira, F.S. Afférri, J.S.S. Rando, \& S.L. Lima. 1994. Efeito da lavagem artificial na eficiência de acaricidas-ovicidas no 
controle de Brevipalpus phoenicis (Geijskes, 1939) (Acari: Tenuipalpidae) em citros. Pesq. Agropec. Bras. 29: 1-5.

Chiavegato, L.G., M.L.B. Trindade, C.E.T. Nogueira \& F.S. Afféri. 1993. Efeito de espalhante adesivo na eficiência de Hexythiazox no controle do ácaro Brevipalpus phoenicis G. (Acari: Tenuipalpidae) em citros. An. Soc. Entomol. Brasil 22:341-348.

Johnstone, D.R. 1973. Spreading and retention of agricultural sprays on foliage. In Van Valkenburg, W. (ed.) Pesticide formulations. New York, Marcel Dekker, 481p.

Martinelli, N.M., C.A.L. de Oliveira \& D. Perecin. 1976. Conhecimentos básicos para estudos que envolvam levantamentos da população do Brevipalpus phoenicis (Geijskes, 1939) na cultura de citros. Científica, 4:242-253.

Matuo, T., S.H. Nakamura \& A. Almeida. 1989. Efeito de alguns adjuvantes da pulverização nas propriedades físicas do líquido. Summa Phytopath. 15:163-173.

Ocampo-Ruiz, R.A. \& T. Matuo. 1994. Efeito de espalhantes-adesivos na retenção e na ação do propargite sobre Brevipalpus phoenicis (Geijs.) em folhas de citros. An. Soc. Entomol. Brasil 23:265-270.

Oliveira, C.A.L. de. 1983. Máquina de varredura de ácaro "Modelo Jaboticabal". An. Soc. Entomol. Brasil 12:299-303.

Recebido em 16/12/96. Aceito em 29/09/97. 